\title{
STABILITY OF TRIGONOMETRIC TYPE FUNCTIONAL EQUATIONS IN RESTRICTED DOMAINS
}

\author{
JAEYOUnG Chung
}

\begin{abstract}
We prove the Hyers-Ulam stability for trigonometric type functional inequalities in restricted domains with time variables. As consequences of the result we obtain asymptotic behaviors of the inequalities and stability of related functional inequalities in almost everywhere sense.
\end{abstract}

\section{INTRODUCTION}

The stability problem of functional equations was originated by S.M. Ulam who released a question concerning the approximate homomorphisms from a group to a metric group (see [26, 27]) and a partial solution was given by D.H. Hyers [11, 10]. After the result of Hyers, T. Aoki [1] and D.G. Bourgin [3, 4] treated with this problem in 1950-1951 and there was no touch of these results until 1978 when Th.M. Rassias [19] treated again with the inequality of Aoki [1]. Following the Rassias' result a great number of papers on the subject have been published concerning numerous functional equations in various directions $[5,6,7,8,9,12,13,14,15,16$, 17, 18, 19, 20, 21, 25]. Among the results, stability problems in restricted domains were investigated by F. Skof [22], S.-M. Jung [12], J.M. Rassias and M.J. Rassias [16]. While studying the stability of trigonometric functional equations [23] in the spaces of distributions [7] one comes across the following functional inequality with time variables

$$
|f(x+y, t+s)-f(x, t) \alpha(y, s)-\alpha(x, t) f(y, s)| \leq \epsilon,
$$

for all $x, y \in \mathbb{R}^{n}, t, s>0$, where $\alpha: \mathbb{R}^{n} \times(0, \infty) \rightarrow \mathbb{C}$ is a bounded exponential function. Let $X$ be a real normed space, $S$ a semigroup, $f: X \times S \rightarrow \mathbb{C}, \epsilon, M>0$

Received by the editors March 4, 2011. Revised May 26, 2011. Accepted June 16, 2011. 2000 Mathematics Subject Classification. 39B82.

Key words and phrases. Hyers-Ulam stability, additive function, exponential function, trigonometric type functional equation, heat kernel. 
and $\Psi: X \times S \rightarrow \mathbb{R}$ a nonnegative function. In this paper we consider the stability of the functional inequality

$$
|f(x+y, t+s)-f(x, t) \alpha(y, s)-\alpha(x, t) f(y, s)| \leq \Psi(x, t)
$$

in the restricted domain $\|x+y\| \geq M$ when $\alpha$ is an unbounded exponential function, and that of the inequality

$$
|f(x+y, t+s)-f(x, t) \alpha(y, s)-\alpha(x, t) f(y, s)| \leq \epsilon
$$

in the restricted domain $\|x+y\| \geq M$ when $\alpha$ is a bounded exponential function. As consequences of the results we obtain the asymptotic behavior of $f$ satisfying

$$
\limsup _{\|x+y\| \rightarrow \infty}|f(x+y, t+s)-f(x, t) \alpha(y, s)-\alpha(x, t) f(y, s)| \leq M
$$

for some positive constant $M$ when $\alpha$ is an unbounded exponential function, and that of $f: X \times S \rightarrow \mathbb{C}$ satisfying

$$
\lim _{\|x+y\| \rightarrow \infty}|f(x+y, t+s)-f(x, t) \alpha(y, s)-\alpha(x, t) f(y, s)|=0
$$

when $\alpha$ is a bounded exponential function. Also we obtain the stability of the inequality

$$
\left|f(x+y)-f(x) e^{c \cdot y}-f(y) e^{c \cdot x}\right| \leq \psi(x)
$$

for almost every $(x, y) \in \mathbb{R}^{2 n}$.

\section{Stability of Trigonometric Type Functional Equations}

Throughout this paper we denote by $G$ a group, $S$ a semigroup, $X$ a real normed space, and $Y$ a Banach space. A function $A: S \rightarrow \mathbb{C}$ is said to be an additive function provided that $A(x+y)=A(x)+A(y)$ for all $x, y \in S$ and $m: S \rightarrow \mathbb{C}$ is said to be an exponential function provided that $m(x+y)=m(x) m(y)$ for all $x, y \in S$. Let $\Psi: X \times S \rightarrow[0, \infty), \epsilon, M \geq 0$ and $f: X \times S \rightarrow \mathbb{C}$. We consider the stability of the functional inequality

$$
|f(x+y, t+s)-f(x, t) \alpha(y, s)-\alpha(x, t) f(y, s)| \leq \Psi(x, t)
$$

for all $x, y \in X, t, s \in S$ with $\|x+y\| \geq M$ when $\alpha: X \times S \rightarrow \mathbb{C}$ is an unbounded exponential function, and that of the functional inequality

$$
|f(x+y, t+s)-f(x, t) \alpha(y, s)-\alpha(x, t) f(y, s)| \leq \epsilon
$$

for all $x, y \in X, t, s \in S$ with $\|x+y\| \geq M$ when $\alpha: X \times S \rightarrow \mathbb{C}$ is a nonzero bounded exponential function. 
Throughout this paper we impose the condition on the semigroup $S$; for any $t_{1}, t_{2} \in S$ there exist a positive integer $k$ and $t_{0} \in S$ such that

$$
k t_{2}=t_{1}+t_{0} .
$$

For example, $S=(0, \infty)$ has the property. We denote $\mathbb{N}$ the set of positive integers.

Lemma 2.1. Let $\alpha: G \times S \rightarrow \mathbb{C}$ be a nonzero exponential function. Then $\alpha(x, t) \neq 0$ for all $x \in G, t \in S$ and $\alpha(x, t)=m_{1}(x) m_{2}(t)$ for all $x \in G, t \in S$, where $m_{1}$ and $m_{2}$ are exponential functions on $G$ and $S$, respectively. In particular, if $\alpha$ is bounded we have

$$
\left|m_{1}(x)\right|=1, \quad\left|m_{2}(t)\right| \leq 1
$$

for all $x \in G, t \in S$.

Proof. If $\alpha\left(x_{1}, t_{1}\right)=0$ for some $x_{1} \in G, t_{1} \in S$, then for given $x \in G, t \in S$, choosing a positive integer $k$ and $t_{0} \in S$ such that $k t=t_{1}+t_{0}$ we have

$$
[\alpha(x, t)]^{k}=\alpha(k x, k t)=\alpha\left(k x-x_{1}, t_{0}\right) \alpha\left(x_{1}, t_{1}\right)=0 .
$$

Since $\alpha$ a nonzero exponential function we have $\alpha(x, t) \neq 0$ for all $x \in G, t \in S$. Let

$$
m(x, t)=\alpha(x, t) \alpha(0, t)^{-1} .
$$

Then we have

$m(x, t)=\alpha(x, t) \alpha(0, t)^{-1}=\alpha(x, t+s) \alpha(0, s)^{-1} \alpha(0, t)^{-1}=\alpha(x, s) \alpha(0, s)^{-1}=m(x, s)$.

Thus $m$ is independent of $t \in S$ and we can write $m(x, t):=m_{1}(x)$. Now we have $m_{1}(x+y)=\alpha(x+y, 2 t) \alpha(0,2 t)^{-1}=m(x, t) \alpha(0, t)^{-1} \alpha(y, t) \alpha(0, t)^{-1}=m_{1}(x) m_{1}(y)$.

Thus $m_{1}$ is an exponential function. Letting $m_{2}(t)=\alpha(0, t)$ we have $\alpha(x, t)=$ $m_{1}(x) m_{2}(t)$. If $\alpha(x, t)=m_{1}(x) m_{2}(t)$ is bounded, there exist $C_{1}, C_{2}>0$ such that $\left|m_{1}(x)\right|^{k}=\left|m_{1}(k x)\right| \leq C_{1}$ for all integers $k$ and $x \in G$, and $\left|m_{2}(t)\right|^{k}=\left|m_{2}(k t)\right| \leq C_{2}$ for all positive integers $k$ and $t \in S$. Thus we have $\left|m_{1}(x)\right|=1$ and $\left|m_{2}(t)\right| \leq 1$ for all $x \in G, t \in S$. This completes the proof.

We first consider the stability of (2.1).

Theorem 2.2. Let $f: X \times S \rightarrow \mathbb{C}$ satisfy (2.1). Then there exists an additive function $A: X \times S \rightarrow \mathbb{C}$ such that

$$
f(x, t)=A(x, t) \alpha(x, t)
$$

for all $x \in X, t \in S$. 
Proof. By Lemma 2.1, $\alpha(x, t) \neq 0$ for all $x \in X, t \in S$. Dividing $|\alpha(x+y, t+s)|$ in both sides of (2.1), letting $A(x, t)=f(x, t) \alpha(x, t)^{-1}$ we have

$$
|A(x+y, t+s)-A(x, t)-A(y, s)| \leq \Psi(x, t)|\alpha(x+y, t+s)|^{-1}
$$

for all $x, y \in X, t, s \in S$ with $\|x+y\| \geq M$. Since $\alpha$ is unbounded we can choose $z_{0} \in X, t_{0}>0$ such that $\left|\alpha\left(z_{0}, t_{0}\right)\right|>1$. For given $x, y \in X, t, s \in S$, choose a positive integer $k_{0}$ such that

$$
\left\|x+y+k z_{0}\right\| \geq M,\left\|y+k z_{0}\right\| \geq M
$$

for all $k \geq k_{0}$. From (2.4), using the triangle inequality we have

$$
\begin{aligned}
\mid A & (x+y, t+s)-A(x, t)-A(y, s) \mid \\
\leq & \left|-A\left(x+y+k z_{0}, t+s+k t_{0}\right)+A(x+y, t+s)+A\left(k z_{0}, k t_{0}\right)\right| \\
& +\left|A\left(x+y+k z_{0}, t+s+k t_{0}\right)-A(x, t)-A\left(y+k z_{0}, s+k t_{0}\right)\right| \\
& +\left|A\left(y+k z_{0}, s+k t_{0}\right)-A(y, s)-A\left(k z_{0}, k t_{0}\right)\right| \\
\leq & (\psi(x+y, t+s)+\psi(x, t))\left|\alpha\left(x+y+k z_{0}, t+s+k t_{0}\right)\right|^{-1} \\
& +\psi(y, s)\left|\alpha\left(y+k z_{0}, s+k t_{0}\right)\right|^{-1} \\
\leq & \left.(\psi(x+y, t+s)+\psi(x, t))|\alpha(x+y, t+s)|^{-1}+\psi(y, s)|\alpha(y, s)|^{-1}\right) \\
& \times\left|\alpha\left(z_{0}, t_{0}\right)\right|^{-k}
\end{aligned}
$$

for all positive integers $k$ with $k \geq k_{0}$. Letting $k \rightarrow \infty$ in (2.5) we have

$$
A(x+y, t+s)-A(x, t)-A(y, s)=0
$$

for all $x, y \in X, t, s \in S$. This completes the proof.

For the stability of (2.2) we need the following.

Lemma 2.3. Let $f: G \times S \rightarrow Y$. Suppose that there exists $\psi: S \times S \rightarrow[0, \infty)$ such that

$$
\|f(x+y, t+s)-f(x, t)-f(y, s)\| \leq \psi(t, s)
$$

for all $x, y \in G, t, s \in S$. Then there exists an additive functions $A: G \rightarrow Y$ such that

$$
\|f(x, 2 t)-A(x)-2 f(0, t)\| \leq 3 \psi(t, t)
$$

for all $x \in G, t \in S$. 
Proof. Replacing $s$ by $t$ and putting $y=0$ in (2.7) we have

$$
\|f(x, 2 t)-f(x, t)-f(0, t)\| \leq \psi(t, t)
$$

for all $x \in G, t \in S$. Replacing $t$ by $s$ and $x$ by $y$ in (2.9) we have

$$
\|f(y, 2 s)-f(0, s)-f(y, s)\| \leq \psi(s, s)
$$

for all $y \in G, s>0$. Using the triangle inequality with the inequalities (2.7), (2.9) and (2.10) we have

$$
\|f(x+y, t+s)-f(x, 2 t)-f(y, 2 s)+f(0, t)+f(0, s)\| \leq \psi(t, s)+\psi(t, t)+\psi(s, s)
$$

for all $x, y \in G, t, s \in S$. Replacing $y$ by $x$ and $s$ by $t$ in (2.11) we have

$$
\|f(2 x, 2 t)-2 f(x, 2 t)+2 f(0, t)\| \leq 3 \psi(t, t)
$$

for all $x \in G, t \in S$. Fixing $t \in S$, replacing $x$ by $2^{k-1} x$ in (2.12) and dividing $2^{k}$ we have

$$
\left\|2^{-k} f\left(2^{k} x, 2 t\right)-2^{-k+1} f\left(2^{k-1} x, 2 t\right)+2^{-k+1} f(0, t)\right\| \leq 3 \cdot 2^{-k} \psi(t, t)
$$

for all $x \in G, t \in S$. For given positive integers $n, m$, putting $k=n, n+1, \ldots, n+m$ in (2.13), summing up the results and using the triangle inequality, we can see that $A_{n}(x, t):=2^{-n} f\left(2^{n} x, 2 t\right), n=1,2,3, \ldots$, is a Cauchy sequence and $A(x, t):=$ $\lim _{n \rightarrow \infty} A_{n}(x, t)$ exists. Replacing $x, y$ by $2^{n} x, 2^{n} y$, respectively in $(2.11)$, diving $2^{n}$ and letting $n \rightarrow \infty$ we have

$$
A(x+y, t+s)-A(x, 2 t)-A(y, 2 s)=0
$$

for all $x, y \in G, t, s \in S$. Letting $x=y=0$ and replacing $s$ by $t$ in (2.14) we have $A(0,2 t)=0$ for all $t \in S$. Thus, putting $y=0$ in (2.14) we have

$$
A(x, t+s)=A(x, 2 t) .
$$

Putting $x=0$ and replacing $y$ by $x$ in (2.14) we have

$$
A(x, t+s)=A(x, 2 s) .
$$

From (2.15) and (2.16) $A(x, 2 t)$ is independent of $t \in S$ and we write $A(x, 2 t):=$ $A(x)$. Putting $k=1,2, \ldots, n$ in $(2.13)$, using the triangle inequality and letting $n \rightarrow \infty$ we have

$$
\|f(x, 2 t)-A(x)-2 f(0, t)\| \leq 3 \psi(t, t)
$$

for all $x \in G, t \in S$. This completes the proof. 
Theorem 2.4. Let $f, \alpha: G \times S \rightarrow \mathbb{C}$ with $\alpha$ a nonzero bounded exponential function. Suppose that there exists nonnegative real numbers $\epsilon$ such that

$$
|f(x+y, t+s)-f(x, t) \alpha(y, s)-\alpha(x, t) f(y, s)| \leq \epsilon
$$

for all $x, y \in G, t, s \in S$. Then there exists an additive functions $A: X \times(0, \infty) \rightarrow \mathbb{C}$ such that

$$
\left|f(x, 2 t)-\left(A(x)+2 f(0, t) \alpha(0, t)^{-1}\right) \alpha(x, 2 t)\right| \leq 3 \epsilon
$$

for all $x \in G, t \in S$.

Proof. Dividing $|\alpha(x+y, t+s)|$ in both sides of (2.18) and letting

$$
g(x, t)=f(x, t) \alpha(x, t)^{-1}
$$

we have

$$
|g(x+y, t+s)-g(x, t)-g(y, s)| \leq \epsilon\left|m_{2}(t+s)\right|^{-1}
$$

for all $x, y \in G, t, s \in S$. Using Lemma 2.3 we have

$$
|g(x, 2 t)-A(x)-2 g(0, t)| \leq 3 \epsilon|\alpha(0,2 t)|^{-1}
$$

for all $x \in G, t \in S$. Multiplying $|\alpha(x, 2 t)|$ in both sides of (2.21) and using Lemma 2.1 we get $(2.19)$. This completes the proof.

Now we prove the stability of the inequality (2.2).

Theorem 2.5. Let $f: X \times S \rightarrow \mathbb{C}$ satisfy (2.2). Then there exists an additive function $A: X \times S \rightarrow \mathbb{C}$ such that

$$
\left|f(x, 2 t)-\left(A(x)+2 f(0, t) \alpha(0, t)^{-1}\right) \alpha(x, 2 t)\right| \leq 3 \epsilon\left(1+2|\alpha(0, t)|^{-1}\right)
$$

for all $x \in X, t \in S$.

Proof. Dividing $|\alpha(x+y, t+s)|$ in both sides of (2.2), using Lemma 2.1 and letting $g(x, t)=f(x, t) \alpha(x, t)^{-1}$ we have

$$
|g(x+y, t+s)-g(x, t)-g(y, s)| \leq \epsilon\left|m_{2}(t+s)\right|^{-1}
$$

for all $x, y \in X, t, s \in S$ with $\|x+y\| \geq M$. For given $x, y \in X, t, s \in S$, choose a $z \in X$ such that

$$
\|x+y+z\| \geq M,\|y+z\| \geq M
$$


Using the triangle inequality and (2.23) we have

$$
\begin{aligned}
&|g(x+y, t+s)-g(x, t)-g(y, s)| \\
& \leq|-g(x+y+z, 2 t+s)+g(x+y, t+s)+g(z, t)| \\
&+|g(x+y+z, 2 t+s)-g(x, t)-g(y+z, s+t)| \\
& \quad+|g(y+z, s+t)-g(y, s)-g(z, t)| \\
& \leq \epsilon\left(2\left|m_{2}(2 t+s)\right|^{-1}+\left|m_{2}(t+s)\right|^{-1}\right)
\end{aligned}
$$

for all $x, y \in X, t, s \in S$. Using Lemma 2.3 we have

$$
|g(x, 2 t)-A(x)-2 g(0, t)| \leq 3 \epsilon\left(2\left|m_{2}(3 t)\right|^{-1}+\left|m_{2}(2 t)\right|^{-1}\right)
$$

for all $x \in X, t \in S$. Multiplying $|\alpha(x, 2 t)|$ in both sides of (2.25) we have

$$
\begin{aligned}
& \left|f(x, 2 t)-\left(A(x)+2 f(0, t) \alpha(0, t)^{-1}\right) \alpha(x, 2 t)\right| \\
& \quad \leq 3 \epsilon\left(2\left|m_{2}(3 t)\right|^{-1}+\left|m_{2}(2 t)\right|^{-1}\right)|\alpha(x, 2 t)| \\
& \quad=3 \epsilon\left(2\left|m_{2}(3 t)\right|^{-1}+\left|m_{2}(2 t)\right|^{-1}\right)\left|m_{2}(2 t)\right| \\
& \quad=3 \epsilon\left(1+2|\alpha(0, t)|^{-1}\right) .
\end{aligned}
$$

This completes the proof.

Corollary 2.6. Let $f, \alpha: X \times(0, \infty) \rightarrow \mathbb{C}$ with $\alpha$ a nonzero bounded exponential function. Suppose that there exist nonnegative real numbers $\epsilon$ and $M$ such that

$$
|f(x+y, t+s)-f(x, t) \alpha(y, s)-\alpha(x, t) f(y, s)| \leq \epsilon
$$

for all $x, y \in X, t, s>0$ with $\|x+y\| \geq M$. Then there exists an additive functions $A: X \times(0, \infty) \rightarrow \mathbb{C}$ such that

$$
\left|f(x, 2 t)-\left(A(x)+2 f(0, t) \alpha(0, t)^{-1}\right) \alpha(x, 2 t)\right| \leq 3 \epsilon(2+\alpha(0, t))
$$

for all $x \in X, t>0$.

Proof. As in the proof of Theorem 2.5, letting $g(x, t)=f(x, t) \alpha(x, t)^{-1}$ we obtain

$$
|g(x+y, t+s)-g(x, t)-g(y, s)| \leq \epsilon\left|m_{2}(t+s)\right|^{-1}
$$

for all $x, y \in X, t, s>0$ with $\|x+y\| \geq M$. For given $x, y \in X, t, s \in S$, choose a $z \in X$ such that

$$
\|x+y+z\| \geq M,\|y+z\| \geq M .
$$


Using the triangle inequality and (2.28) we have

$$
\begin{aligned}
&|g(x+y, t+s)-g(x, t)-g(y, s)| \\
& \leq|-g(x+y+z,(1+r) t+s)+g(x+y, t+s)+g(z, r t)| \\
& \quad+|g(x+y+z,(1+r) t+s)-g(x, t)-g(y+z, s+r t)| \\
& \quad+|g(y+z, s+r t)-g(y, s)-g(z, r t)| \\
& \leq \epsilon\left(2\left|m_{2}((1+r) t+s)\right|^{-1}+\left|m_{2}(r t+s)\right|^{-1}\right) \\
&:= \psi_{r}(t, s) .
\end{aligned}
$$

for all $x, y \in X, t, s, r>0$. Using Lemma 2.3 we have

$$
|g(x, 2 t)-A(x)-2 g(0, t)| \leq 3 \psi_{r}(t, t)
$$

for all $x \in X, t>0$ and $r>0$. Multiplying $|\alpha(x, 2 t)|$ in both sides of (2.30) we have

$$
\begin{aligned}
& \left|f(x, 2 t)-\left(A(x)+2 f(0, t) \alpha(0, t)^{-1}\right) \alpha(x, 2 t)\right| \\
& \quad \leq 3 \psi_{r}(t, t)|\alpha(x, 2 t)| \\
& \quad=3 \psi_{r}(t, t)\left|m_{2}(2 t)\right| \\
& \quad \leq 3 \epsilon\left|m_{2}(r t)\right|^{-1}\left(2+m_{2}(t)\right) .
\end{aligned}
$$

In view of Lemma 2.1 , for each $t>s>0$ we have

$$
\left|m_{2}(t)\right|^{-1}=\left|m_{2}(s)\right|^{-1}\left|m_{2}(t-s)\right|^{-1} \geq\left|m_{2}(s)\right|^{-1} \geq 1 .
$$

Thus $\left|m_{2}(t)\right|^{-1}$ is decreasing as $t \rightarrow 0^{+}$and hence $\lim _{t \rightarrow 0}\left|m_{2}(t)\right|:=\gamma$ exists. Now $\gamma=\lim _{t \rightarrow 0}\left|m_{2}(t)\right|=\lim _{t \rightarrow 0}\left|m_{2}(t / 2)\right|^{2}=\gamma^{2}$, which implies $\gamma=1$. Letting $r \rightarrow 0^{+}$ in the right hand side of (2.31) we get (2.27). This completes the proof.

\section{Asymptotic Behaviors}

In this section we consider asymptotic behavior of $f: X \times S \rightarrow \mathbb{C}$ satisfying

$$
\limsup _{\|x+y\| \rightarrow \infty}|f(x+y, t+s)-f(x, t) \alpha(y, s)-\alpha(x, t) f(y, s)| \leq M
$$

for some positive constant $M$ when $\alpha$ is an unbounded exponential function, and that of $f: X \times S \rightarrow \mathbb{C}$ satisfying

$$
\lim _{\|x+y\| \rightarrow \infty}|f(x+y, t+s)-f(x, t) \alpha(y, s)-\alpha(x, t) f(y, s)|=0
$$

when $\alpha$ is bounded exponential.

Using the idea of Theorem 2.2 we have the following. 
Theorem 3.1. Let $f: X \times S \rightarrow \mathbb{C}$ satisfy (3.1). Then there exists an additive function $A: X \times S \rightarrow \mathbb{C}$ such that

$$
f(x, t)=A(x, t) \alpha(x, t)
$$

for all $x \in X, t \in S$.

Proof. From the condition (3.1) there exists $d>0$ such that

$$
|f(x+y, t+s)-f(x, t) \alpha(y, s)-\alpha(x, t) f(y, s)| \leq M+1
$$

for all $x, y \in X, t, s \in S$ with $\|x+y\| \geq d$. Now using Theorem 2.2 we obtain that

$$
A(x+y, t+s)-A(x, t)-A(y, s)=0
$$

for all $x, y \in X, t, s \in S$, where $A(x, t)=f(x, t) \alpha(x, t)^{-1}$. This completes the proof.

Remark. The author guesses that the Theorem 3.1 can be refined as follows: If $f: X \times S \rightarrow \mathbb{C}$ satisfies

$$
\limsup _{\|y\| \rightarrow \infty}|f(x+y, t+s)-f(x, t) \alpha(y, s)-\alpha(x, t) f(y, s)| \leq \Psi(x, t, s)
$$

for some nonnegative function $\Psi(x, t, s)$, then $f$ has the form (3.3).

Using Theorem 2.5 we obtain the following.

Theorem 3.2. Let $f: X \times S \rightarrow \mathbb{C}$ satisfy (3.2). Then there exists an additive functions $A: X \times S \rightarrow \mathbb{C}$ such that

$$
f(x, 2 t)=\left(A(x)+2 f(0, t) \alpha(0, t)^{-1}\right) \alpha(x, 2 t)
$$

for all $x \in X, t \in S$.

Proof. From the condition (3.2), for each positive integers $n$ there exists $d_{n}>0$ such that

$$
|f(x+y, t+s)-f(x, t) \alpha(y, s)-\alpha(x, t) f(y, s)| \leq \frac{1}{n}
$$

for all $x, y \in X, t, s \in S$ with $\|x+y\| \geq d_{n}$. By Theorem 2.5, there exist additive functions $A_{n}$ such that

$$
\left|f(x, 2 t)-\left(A_{n}(x)+2 f(0, t) \alpha(0, t)^{-1}\right) \alpha(x, 2 t)\right| \leq \frac{3}{n}\left(1+2|\alpha(0, t)|^{-1}\right)
$$

for all $x \in X, t \in S$. Replacing $n$ by another positive integer $m$ in (3.9) and using the triangle inequality we have

$$
\left|A_{n}(x)-A_{m}(x)\right||\alpha(x, 2 t)| \leq 6\left(1+2|\alpha(0, t)|^{-1}\right)
$$


for all $x \in X, t \in S$. Dividing $|\alpha(x, 2 t)|$ in both sides of (3.10) and using Lemma 2.1 we have

$$
\left|A_{n}(x)-A_{m}(x)\right| \leq 6\left|m_{2}(2 t)\right|^{-1}\left(1+2|\alpha(0, t)|^{-1}\right)
$$

for all $x \in X, t \in S$, where $m_{2}$ is an exponential function on $S$. Since $A$ is additive we have

$$
\begin{aligned}
\left|A_{n}(x)-A_{m}(x)\right| & \leq \frac{1}{k}\left|A_{n}(k x)-A_{m}(k x)\right| \\
& \leq \frac{6}{k}\left|m_{2}(2 t)\right|^{-1}\left(1+2|\alpha(0, t)|^{-1}\right)
\end{aligned}
$$

for all $x \in X, t \in S$ and positive integers $k$. Letting $k \rightarrow \infty$ in (3.12) we have $A_{n}=A_{m}$ for all positive integers $n, m$. Finally, letting $n \rightarrow \infty$ in (3.9) we get the result.

\section{Stabilities in Almost Everywhere Sense}

Let $f$ be a Lebesgue measurable function on $\mathbb{R}^{n}$ satisfying the inequality; for every $\epsilon>0$ there exists $C_{\epsilon}>0$ such that

$$
|f(x)| \leq C_{\epsilon} e^{\epsilon|x|^{2}}
$$

for all $x \in \mathbb{R}^{n}$. The function satisfying (4.1) is said to be an infra-exponential function of order 2 . We denote by $E_{t}$ be the $n$-dimensional heat kernel[28] given by,

$$
E_{t}(x)=(4 \pi t)^{-\frac{n}{2}} e^{\frac{-|x|^{2}}{4 t}}, \quad x \in \mathbb{R}^{n}, t>0 .
$$

For every infra-exponential function $f$ of order 2 , the convolution with the heat kernel

$$
F(x, t):=f * E_{t}(x)=\int f(x-y) E_{t}(y) d y
$$

is well defined and is a smooth solution of the heat equation $\left(\partial / \partial_{t}-\Delta\right) F=0$ in $\left\{(x, t): x \in \mathbb{R}^{n}, t>0\right\}$ and $f * E_{t}(x) \rightarrow f(x)$ as $t \rightarrow 0^{+}$for almost every $x \in \mathbb{R}^{n}$. We call $f * E_{t}(x)$ the Gauss transform of $f$. From now on we denote by $\Omega$ a subset of $\mathbb{R}^{2 n}$ with the Lebesgue measure $m\left(\mathbb{R}^{2 n} \backslash \Omega\right)=0$.

Theorem 4.1. Let $c=\left(c_{1}, \ldots, c_{n}\right) \in \mathbb{C}^{n}$ with $\Re c_{j} \neq 0$ for some $j=1,2, \ldots, n$ and $\psi: \mathbb{R}^{n} \rightarrow[0, \infty)$ an infra-exponential function of order 2 . Suppose that $f: \mathbb{R}^{n} \rightarrow \mathbb{C}$ be an infra-exponential function of order 2 satisfying

$$
\left|f(x+y)-f(x) e^{c \cdot y}-f(y) e^{c \cdot x}\right| \leq \psi(x)
$$


for all $(x, y) \in \Omega$. Then there exists $a \in \mathbb{C}^{n}$ such that

$$
f(x)=a \cdot x e^{c \cdot x}
$$

for almost every $x \in \mathbb{R}^{n}$.

Proof. Multiplying $E_{t}(\xi-x) E_{s}(\eta-y)$ in both sides of (4.2) and integrating the result with respect to $x$ and $y$ we have

$$
\left|F(\xi+\eta, t+s)-F(\xi, t) e^{c \cdot \eta+\left(c_{1}^{2}+\ldots+c_{n}^{2}\right) s}-F(\eta, s) e^{c \cdot \xi+\left(c_{1}^{2}+\ldots+c_{n}^{2}\right) t}\right| \leq \Psi(\xi, t)
$$

for all $\xi, \eta \in \mathbb{R}^{n}$, where

$$
F(\xi, t)=\int f(z) E_{t}(\xi-z) d z, \quad \Psi(\xi, t)=\int \psi(z) E_{t}(\xi-z) d z .
$$

Using Theorem 2.2 we have

$$
F(\xi, t)=A(\xi, t) e^{c \cdot \xi+\left(c_{1}^{2}+\ldots+c_{n}^{2}\right) t}
$$

for all $\xi, \eta \in \mathbb{R}^{n}, t, s>0$, where $A(\xi, t)$ is an additive function. It follows from the continuity of $F$ that $A(\xi, t)$ is a Lebesgue measurable solution and the solution $A$ of the Cauchy functional equation

$$
A(\xi+\eta, t+s)=A(\xi, t)+A(\eta, s)
$$

is given by

$$
A(\xi, t)=a \cdot \xi+b t, \quad a \in \mathbb{C}^{n}, b \in \mathbb{C} .
$$

Thus we have

$$
F(\xi, t)=(a \cdot \xi+b t) e^{c \cdot \xi+\left(c_{1}^{2}+\ldots+c_{n}^{2}\right) t} .
$$

Letting $t \rightarrow 0^{+}$in (4.5) we get the result.

Theorem 4.2. Let $c=\left(c_{1}, \ldots, c_{n}\right) \in \mathbb{R}^{n}$. Suppose that $f: \mathbb{R}^{n} \rightarrow \mathbb{C}$ is an infraexponential function of order 2 satisfying

$$
\left|f(x+y)-f(x) e^{i c \cdot y}-f(y) e^{i c \cdot x}\right| \leq \epsilon
$$

for all $(x, y) \in \Omega$. Then there exist $a \in \mathbb{C}^{n}, b \in \mathbb{C}$ such that

$$
\left|f(x)-(a \cdot x+b) e^{i c \cdot x}\right| \leq 3 \epsilon
$$

for almost every $x \in \mathbb{R}^{n}$. 
Proof. Multiplying $E_{t}(\xi-x) E_{s}(\eta-y)$ in both sides of (4.6) and integrating the result with respect to $x$ and $y$ we have

$$
\left|F(\xi+\eta, t+s)-F(\xi, t) e^{i c \cdot \eta-\left(c_{1}^{2}+\ldots+c_{n}^{2}\right) s}-F(\eta, s) e^{i c \cdot \xi-\left(c_{1}^{2}+\ldots+c_{n}^{2}\right) t}\right| \leq \epsilon
$$

for all $\xi, \eta \in \mathbb{R}^{n}, t, s>0$, where

$$
F(\xi, t)=\int f(z) E_{t}(\xi-z) d z .
$$

Using Theorem 2.4 we have

$$
\left|F(\xi, t)-(A(\xi)+2 F(0, t / 2)) e^{i c \cdot \xi-\left(c_{1}^{2}+\ldots+c_{n}^{2}\right) t}\right| \leq 3 \epsilon
$$

for all $\xi, \eta \in \mathbb{R}^{n}, t>0$. In view of the proof of Lemma 2.3, the continuity of $F$ implies $A(\xi)=a \cdot \xi$ for some $a \in \mathbb{C}^{n}$. Putting $\xi=\eta=0$ in (4.8), multiplying $e^{\left(c_{1}^{2}+\ldots+c_{n}^{2}\right) s}$ and using the triangle inequality we have

$$
|F(0, t)| \leq e^{\left(c_{1}^{2}+\ldots+c_{n}^{2}\right) s}\left(\left|F(0, t+s)-F(0, s) e^{\left(c_{1}^{2}+\ldots+c_{n}^{2}\right) t}\right|+\epsilon\right)
$$

for all $t, s>0$. In view of (4.10), it follows from the continuity of $F$ that

$$
\limsup _{t \rightarrow 0^{+}} F(0, t):=b / 2
$$

exists. Also it is well known that $F(\xi, t) \rightarrow f(\xi)$ as $t \rightarrow 0^{+}$for almost every $\xi \in \mathbb{R}^{n}$. Letting $t \rightarrow 0^{+}$in $(4.5)$ so that $F(0, t / 2) \rightarrow b / 2$ we get the result. This completes the proof.

\section{ACKNOWLEDGMENT}

The author was supported by the Korea Research Foundation Grant (KRF) grant funded by the Korea Government (MEST)(No. 2010-0016963).

\section{REFERENCES}

1. T. Aoki: On the stability of the linear transformation in Banach spaces. J. Math. Soc. Japan 2 (1950), 64-66.

2. J.A. Baker: The stability of cosine functional equation. Proc. Amer. Math. Soc. 80 (1980) 411-416.

3. D.G. Bourgin: Class of transformations and bordering transformations. Bull. Amer. Math. Soc. 57 (1951), 223-237.

4. __ Multiplicative transformations. Proc. Nat. Academy Sci. of U.S.A. 36 (1950), $564-570$. 
5. J. Chung: Stability of approximately quadratic Schwartz distributions. Nonlinear Analysis 67 (2007) 175-186.

6. $\quad$ : A distributional version of functional equations and their stabilities. Nonlinear Analysis 62 (2005), 1037-1051.

7. J. Chang \& J. Chung: The stability of the sine and cosine functional equations in Schwartz distributions. Bull. Korean Math. Soc. 45 (2009), no. 1, 87-97.

8. S. Czerwik: Stability of Functional Equations of Ulam-Hyers-Rassias Type. Hadronic Press, Inc., Palm Harbor, Florida, Florida,2003.

9. G.L. Forti: Hyer-Ulam stability of functional equation in several variables. Aequationes Math. 50 (1995), 143-190.

10. D.H. Hyers: On the stability of the linear functional equations. Proc. Nat. Acad. Sci. USA 27 (1941), 222-224.

11. D.H. Hyers, G. Isac \& Th.M. Rassias: Stability of functional equations in several variables. Birkhauser, 1998.

12. S.-M. Jung: Hyers-Ulam stability of Jensen's equation and its application Proc. Amer. Math. Soc. 126 (1998), 3137-3143.

13. K.-W. Jun \& H.-M. Kim: Stability problem for Jensen-type functional equations of cubic mappings. Acta Mathematica Sinica, English Series 22 (2006), no. 6, 1781-1788.

14. G.H. Kim \& Y.-H. Lee: Boundedness of approximate trigonometric functional equations. Appled Mathematics Letters 31 (2009), 439-443.

15. C.G. Park: Hyers-Ulam-Rassias stability of homomorphisms in quasi-Banach algabras. Bull. Sci. Math. 132 (2008), 87-96.

16. J.M. Rassias \& M.J. Rassias: On the Ulam stability of Jensen and Jensen type mappings on restricted domains. J. Math. Anal. Appl. 281 (2003), 516-524.

17. J.M. Rassias: On the Ulam stability of mixed type mappings on restricted domains. $J$. Math. Anal. Appl. 276 (2002), 747-762.

18. __ : On Approximation of Approximately Linear Mappings by Linear Mappings. J. Funct. Anal. 46 (1982), 126-130.

19. Th.M. Rassias: On the stability of functional equations in Banach spaces J. Math. Anal. Appl. 251 (2000), 264-284.

20. __ On the stability of the linear mapping in Banach spaces. Proc. Amer. Math. Soc. 7 (1978), 297-300.

21. K. Ravi \& M. Arunkumar: On the Ulam-Gavruta-Rassias stability of the orthogonally Euler-Lagrange type functional equation. Intern. J. Appl. Math. Stat. 7 (2007), 143-156.

22. F. Skof: Sull'approssimazione delle applicazioni localmente $\delta$-additive. Atii Accad. Sci.Torino Cl. Sci. Fis. Mat. Natur. 117 (1983), 377-389.

23. L. Székelyhidi: The stability of sine and cosine functional equations. Proc. Amer. Math. Soc. 110 (1990), 109-115. 
24. : The stability of d'Alembert type functional equations. Acta Sci. Math. Szeged. 44 (1982c), 313-320.

25. I. Tyrala: The stability of d'Alembert's functional equation. Aequationes Math. 69 (2005), 250-256.

26. S.M. Ulam: Problems in modern mathematics. Interscience Publ., New York, 1960.

27. : A Collection of Mathematical Problems. Science Editions, Wiley, 1968.

28. D.V. Widder: The heat equation. Academic Press, New York, 1975.

Department of Mathematics, Kunsan National University, Kunsan 573-701, Republic OF KOREA

Email address: jychung@kunsan.ac.kr 